\title{
Effect of Nanoscale Heating on Electrical Transport in RF MEMS Switch Contacts
}

\author{
Brian D. Jensen, Linda L.-W. Chow, Student Member, IEEE, Kuangwei Huang, Kazuhiro Saitou, Member, IEEE, \\ John L. Volakis, Fellow, IEEE, and Katsuo Kurabayashi, Member, IEEE
}

\begin{abstract}
This paper explores contact heating in microelectromechanical systems (MEMS) switches with contact spot sizes less than $100 \mathrm{~nm}$ in diameter. Experiments are conducted to demonstrate that contact heating causes a drop in contact resistance. However, existing theory is shown to over-predict heating for MEMS switch contacts because it does not consider ballistic transport of electrons in the contact. Therefore, we extend the theory and develop a predictive model that shows excellent agreement with the experimental results. It is also observed that mechanical cycling causes an increase in contact resistance. We identify this effect as related to the build-up of an insulating film and demonstrate operational conditions to prevent an increase in contact resistance. The improved understanding of contact behavior gained through our modeling and experiments allows switch performance to be improved.

[1424]
\end{abstract}

Index Terms-Electrical contacts, microelectromechanical systems (MEMS), switches.

\section{INTRODUCTION}

$\mathbf{M}$ ANY examples of metal contact RF MEMS switches are presented in the literature (for example, [1]-[3]). These have demonstrated the excellent performance typical of MEMS switches-high off-state impedance, low on-state impedance, excellent linearity, and low power consumption. This performance makes them attractive alternatives to solid-state switches in military and commercial radar systems, satellite and wireless communications systems, and wireless sensors [4]. In addition to the advantages mentioned above, metal contact switches offer significantly wider bandwidth as compared to capacitive switches, allowing them to be used in reconfigurable antennas and circuits intended for multiple frequency bands. Understanding the failure of metal contact switches is challenging, however, due to the complex interactions between deformation, current flow, and heating at the contact [5]. Still, individual examples of such switches have demonstrated reliable operation to several billion cycles [3], [6].

Several failure mechanisms have been noted for metal contact switches, including adhesion [3], melting, material transport [7], thermally induced explosions related to boiling of the contact

Manuscript received September 8, 2004; revised February 15, 2005. This work was supported by the National Science Foundation, a National Defense Science and Engineering Fellowship, and the CIA. Subject Editor H. Fujita.

B. D. Jensen is with the Department of Mechanical Engineering, Brigham Young University, Provo, UT 84602 USA (e-mail: bdjensen@byu.edu).

L. L.-W. Chow, K. Huang, K. Saitou, and K. Kurabayashi are with the Department of Mechanical Engineering, University of Michigan, Ann Arbor, MI 48109 USA (e-mail: lindchow@umich.edu; kuangwei@umich.edu; kazu@umich.edu; katsuo@umich.edu).

J. L. Volakis is with the Department of Electrical and Computer Engineering, The Ohio State University, Columbus, OH 43212 USA (e-mail: volakis@ ece.osu.edu)

Digital Object Identifier 10.1109/JMEMS.2005.856653 metal [8], and increasing contact resistance [1], [3]. Typically, these failure mechanisms have been noted rather than studied in more depth. For example, an increase in contact resistance as the switch cycles has been reported for both large-scale silver contacts [9] and for MEMS contacts at high cycle numbers [1], [3], but these works have not suggested a hypothesis for the physical cause of the increase.

In addition, it is significant that each failure mechanism above relates to the contact behavior. Therefore, improvements in reliability and power handling capability require a solid understanding of the physics governing contact formation. However, while contact mechanics has been studied extensively over many years, microscale contacts present new challenges. The forces in MEMS contacts (typically tens to hundreds of micro-Newtons) are approximately one thousand times smaller than what has previously been considered as a microcontact force-on the order of $200 \mathrm{mN}$ [10]. Such small forces produce contact spots with size normally comparable to or smaller than the electron mean free path in the material $(<50 \mathrm{~nm})$, leading to additional contact resistance due to boundary scattering of electrons passing though the contact.

Heating in contacts due to the passage of current has been studied extensively by Holm [10]. Moreover, finite difference analysis [11] and finite element analysis [12] have both been applied to the heating of contacts in MEMS switches. In all of these works, however, the contact spot size has been assumed to be larger than the electron mean free path. Our experiments suggest that MEMS contacts frequently have spot sizes on the order of or smaller than the mean free path. Such small contact spots are expected to experience reduced heating compared to spots larger than the mean free path.

Hence, in this paper we show that existing contact theory significantly over-predicts contact heating for such small contact spots. We develop improved theory of contact heating for small spots and demonstrate the use of heating for preventing contact resistance increase. In multiple experimental results, we show the accuracy of the improved theory. Further, we investigate the causes of contact resistance increase. The resulting understanding allows improved switch operation by keeping resistance low.

\section{EXISTING CONTACT THEORY}

A significant component of contact resistance is caused by the roughness of the contacting surfaces (see Fig. 1). As the surfaces come together, high points on each surface make contact, producing real contact at a finite number of asperities. MEMS switch contacts operated under typical conditions are also likely to be covered by a thin insulating film due to process residuals, impurities in the ambient (most likely hydrocarbons), or some 
other source. The presence of this insulating film further limits metal-to-metal contact by allowing real contact only at breaks in the film. This creates a restriction causing a larger resistance across the contact than results from contact asperities alone.

A contact normally consists of multiple spots of different sizes. Therefore, prediction of contact spot size distribution is necessary to fully model contact resistance. Assuming that the spots are sufficiently far apart that they do not interfere significantly with each other, the resistance of the $i$ th spot, $r_{c i}$, acts in parallel with the others. Hence, the total contact resistance $R_{c}$, accounting for $N$ spots, is given by

$$
R_{c}=\left(\sum_{i=1}^{N} r_{c i}^{-1}\right)^{-1} .
$$

Contact surfaces are often modeled using fractal theory, where the contact spot distribution follows the power law proposed by Mandelbrot [13]. Integrating over each spot gives [14]

$$
A_{T}=\frac{D-1}{3-D} A_{L}
$$

where $A_{T}$ is the total contact area, $D$ is the fractal dimension (a parameter between 2 and 3 ), and $A_{L}$ is the area of the largest contact spot. AFM imaging of the gold surfaces used for our experiments showed that $D$ for this case is less than 2.05, and therefore $A_{T}$ is approximately equal to $A_{L}$-indicating that the largest spot $\left(i=N_{L}\right)$, with the lowest contact resistance, dominates the total contact resistance. Then

$$
R_{c} \approx r_{c N_{L}}
$$

Therefore, the contact resistance can be treated as if it were caused by a single contact spot. By contrast, some previous work in MEMS contacts has estimated that a few tens of asperities were in contact [1], [15]. However, this previous work used a different fabrication process which resulted in much rougher contact dimples, which would cause the assumption that $D$ is near 2 to be violated. Moreover, the estimate that one asperity dominates the resistance does not preclude the presence of additional contact asperities, so long as the additional contact spots are sufficiently smaller than the largest spot to contribute little to the contact conductance.

As current flows, it heats the contact spot to an elevated temperature. This heating can be extremely localized, resulting in contact temperatures tens or even hundreds of degrees higher than the surrounding material. For metal contacts, Holm has expressed the contact spot temperature $T_{c}$ as a function of contact voltage $V_{c}$ as [10]

$$
T_{c}=\sqrt{\frac{V_{c}^{2}}{4 L}+T_{0}^{2}} .
$$

Here, $L=2.45 \times 10^{-} 8 \mathrm{~W} \cdot \Omega / \mathrm{K}^{2}$ is the Lorentz constant and $T_{0}$ is the ambient temperature. At sufficient contact temperature, annealing of the contact takes place, reducing the contact hardness (a phenomenon known as "contact softening" [10]). Moreover, heating of the contact spot may also cause breakdown or delamination of the insulating film, allowing real contact over a larger area. Either effect is measured as a decrease in contact resistance. This is illustrated in Fig. 1. The published softening

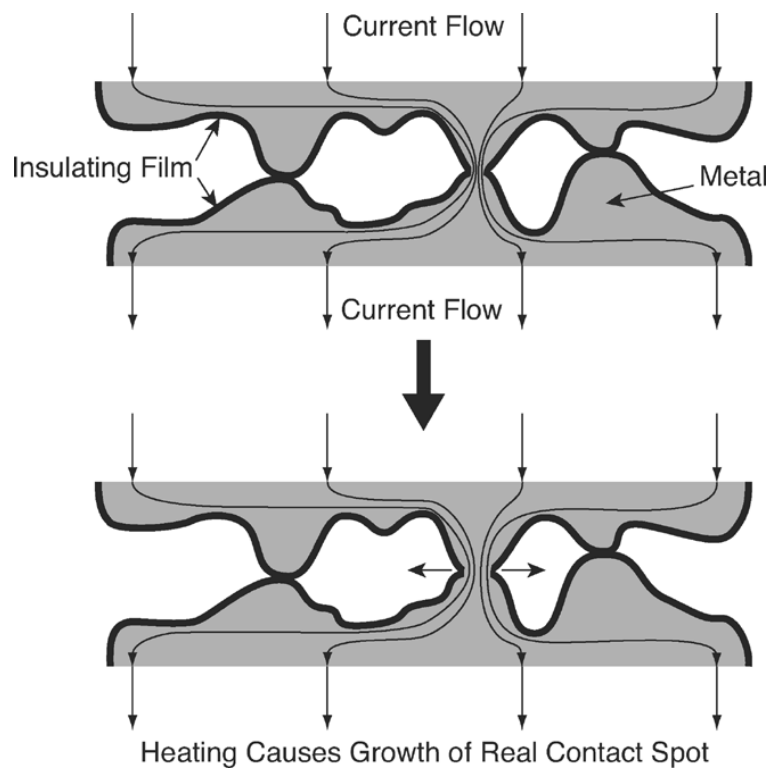

Fig. 1. Illustration of contribution of surface roughness and insulating films to contact resistance. Heating due to current flow in the contact spot leads to a resistance decrease caused by material annealing or film breakdown.

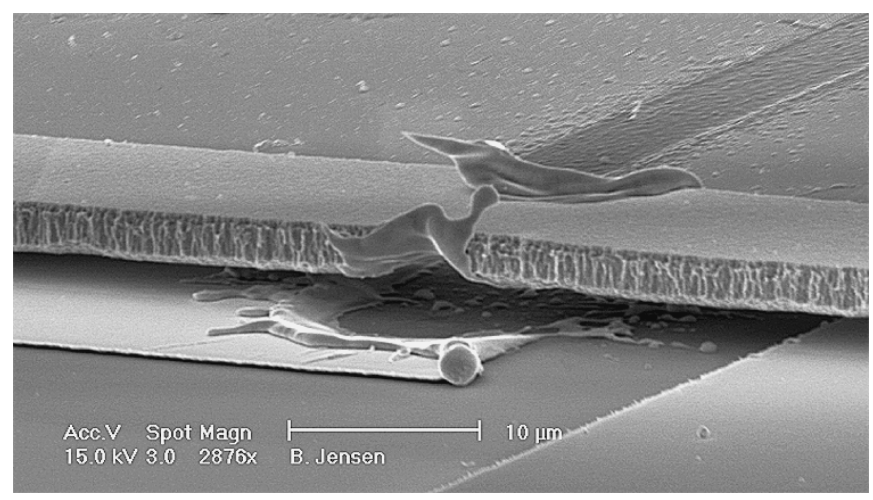

Fig. 2. SEM of a gold contact which boiled due to current flow.

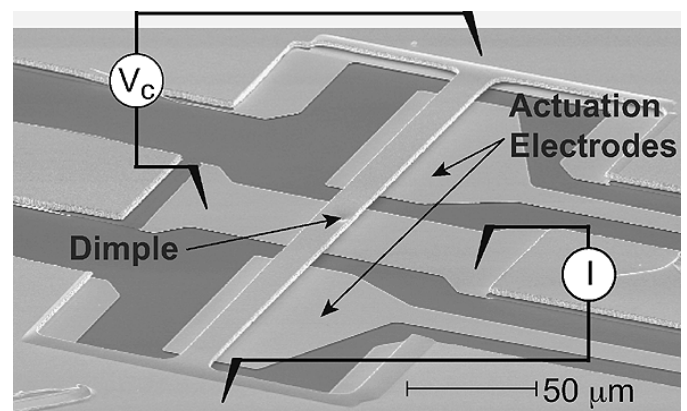

Fig. 3. SEM image of a sample contact-type switch. Probe placement is shown for four-point-probe measurement of contact resistance.

temperature for gold contacts is $100^{\circ} \mathrm{C}$, corresponding to a contact voltage of $70-80 \mathrm{mV}$ for contacts near room temperature [10]. Contact melting or boiling (see Fig. 2) is also possible at higher temperatures. For gold these occur at 1063 and $2817^{\circ} \mathrm{C}$, respectively, or about 430 and $900 \mathrm{mV}$, according to (4).

\section{FABRICATION AND EXPERIMENTS}

To study contact heating and its effects on contact resistance, we fabricated and tested metal-contact MEMS switches. A SEM 


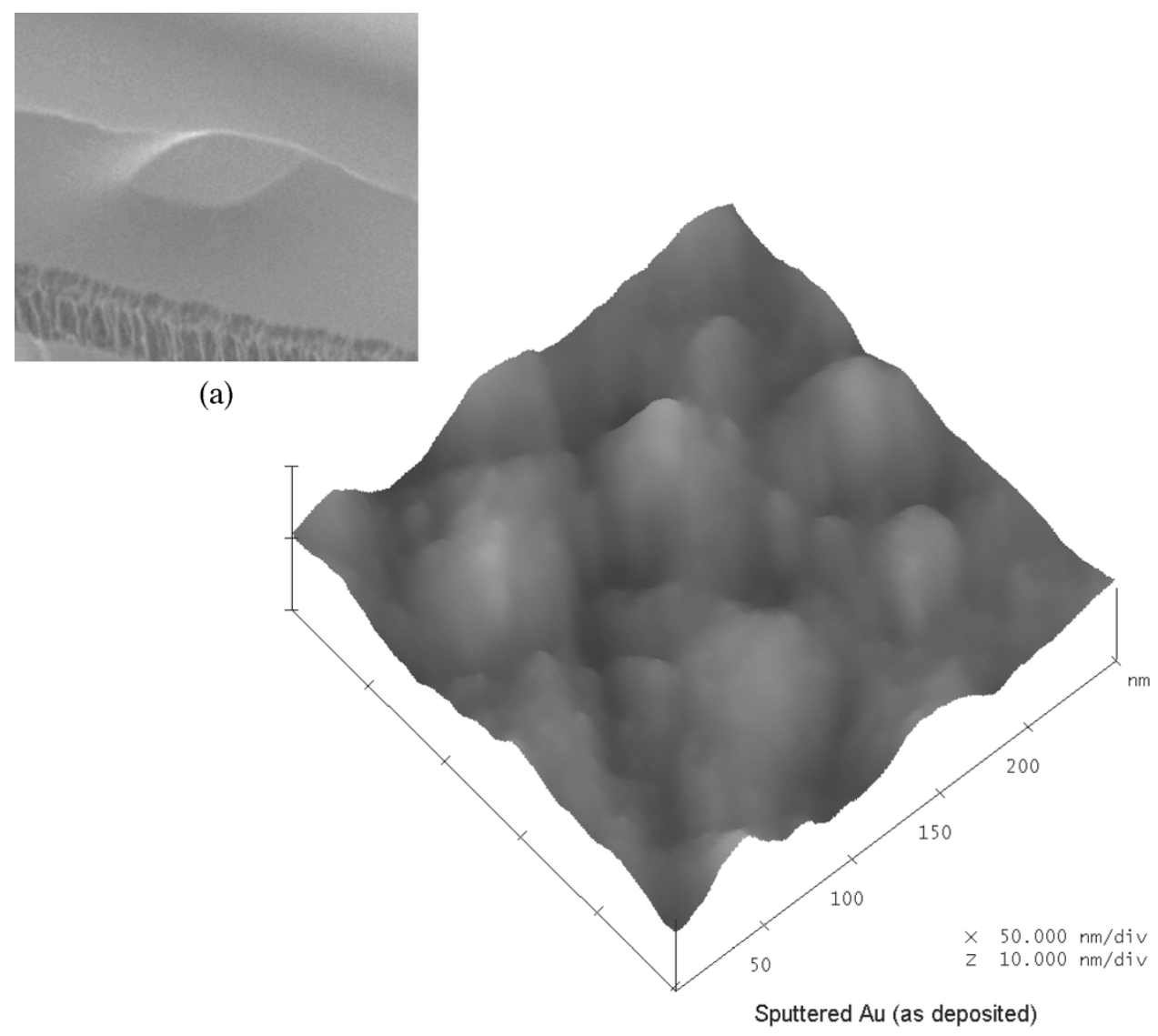

(b)

Fig. 4. (a) SEM image of the micromachined dimple, and (b) AFM scan of the gold contact surface.

image of a typical switch is shown in Fig. 3. The switch consists of a fixed-fixed beam situated across the ground lines of a coplanar waveguide. The beam and underlying electrodes are sputtered gold. Electrostatic force is used to pull the beam down until the dimple in the center of the beam contacts the central conductor of the waveguide. To avoid charging, there is no dielectric film coating the actuation electrodes. Instead, the stiffness of the beam is relied upon to prevent shorting. The total gap under the beam is $1.54 \mu \mathrm{m}$, with a dimple height of $1.18 \mu \mathrm{m}$, leaving a distance of $0.36 \mu \mathrm{m}$ to travel before contact occurs. The tested beams had a width of $100 \mu \mathrm{m}$, a thickness of $3.1 \mu \mathrm{m}$, and a length of either 400 or $500 \mu \mathrm{m}$. The beam geometry allows four-point probe measurements of contact resistance, as illustrated in Fig. 3. The dimples varied in size between $5 \times 5$ and $20 \times 20 \mu \mathrm{m}$, but no difference in contact resistance behavior was seen between dimples of varying size. SEM imaging of the dimples suggests that the bottoms are very flat, without any detectable curvature, as shown in Fig. 4(a). Similarly, AFM imaging of the contact electrode showed that it has rms roughness of approximately $13 \mathrm{~nm}$. A sample AFM scan is shown in Fig. 4(b).

We measured switch pull-down voltage (the voltage just required to initiate contact) and ultimate pull-in voltage (the voltage causing unstable collapse onto the actuation electrodes) and compared them to predictions of a mechanical model to extract Young's modulus and residual stress. We estimated the Young's modulus of our gold film to be $50 \pm 5 \mathrm{GPa}$, with a residual tensile stress of about $92 \pm 6 \mathrm{MPa}$. Several papers have

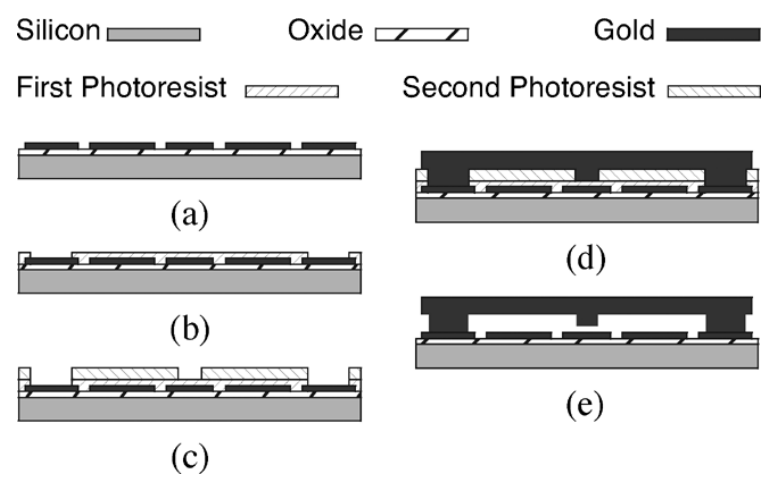

Fig. 5. Illustration of fabrication steps.

previously reported estimates between 50-55 GPa for Young's modulus of microfabricated gold structures, comparing well with our measurement [16]-[18]. Contact occurred at approximately $55 \mathrm{~V}$ for the $500 \mu \mathrm{m}$ beam and about $60 \mathrm{~V}$ for the $400 \mu \mathrm{m}$ beam, and catastrophic collapse onto the actuation electrodes occurs at about 100 and $124 \mathrm{~V}$, respectively.

The contact force in the switches was calculated based on the measured actuation voltage using a mechanical-electrostatic model employing the finite difference method to simulate mechanical deflection. A reduced-order model based on relations for the capacitance of a microstrip line was used to simulate the electrostatic force [19]. The model was validated by comparison to simulations using both ANSYS and CoventorWare. 


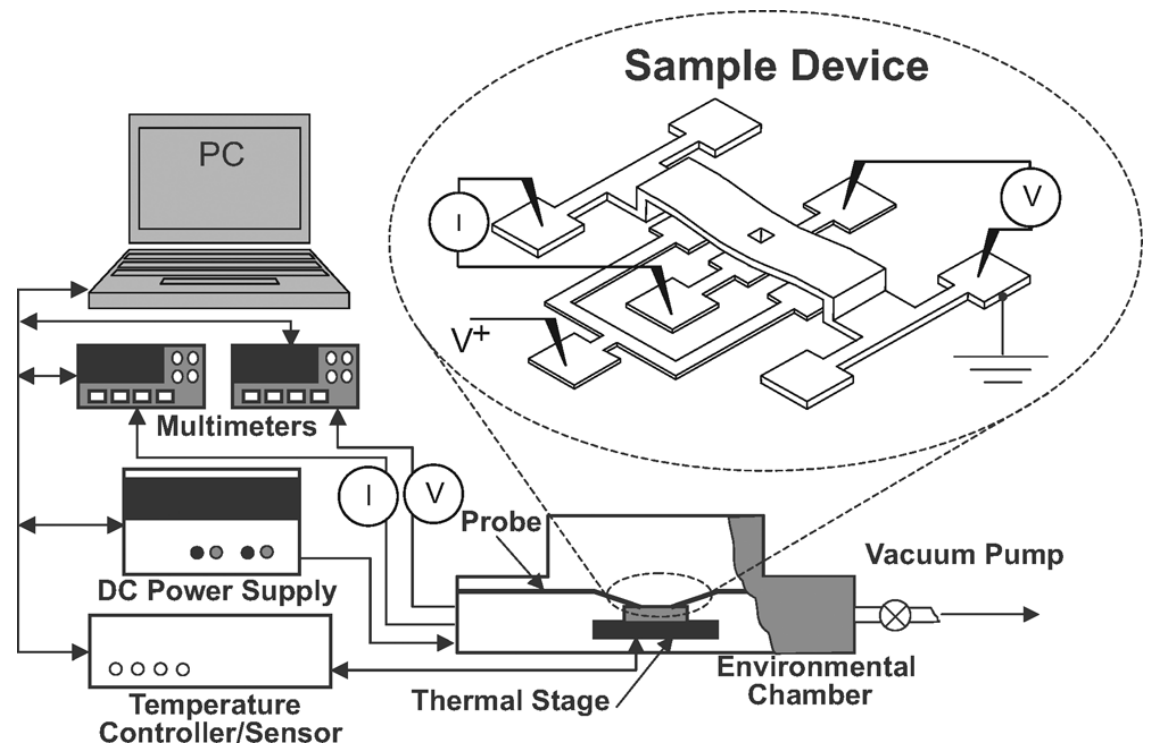

Fig. 6. Experimental setup.

Both comparisons showed a maximum error in the contact force of less than $1.4 \%$ over a variety of loading conditions. However, the uncertainty in the values of Young's modulus and residual stress contributes to uncertainty in the contact force predictions. Overall, we estimate that the contact forces reported here are accurate to within $\pm 10 \mu \mathrm{N}$ up to a contact force of about $218 \mu \mathrm{N}$ [20]. Using this same technique, we estimate contact opening forces (or elastic restoring forces) of 60 and $70 \mu \mathrm{N}$ for the 500 and $400 \mu \mathrm{m}$ beams, respectively.

\section{A. Fabrication}

The switches were fabricated using metal surface micromachining, shown in Fig. 5. The substrate is a silicon wafer with a layer of thermal oxide for isolation. The first gold layer, used for actuation and contact electrodes and for wiring, is sputtered and patterned, Fig. 5(a). Next, a thin layer of photoresist is spun on and patterned to define the anchors, Fig. 5(b). The thickness of this layer determines the gap between the dimple and the contact electrode. The second layer of photoresist is then spun on and patterned to define the dimples, Fig. 5(c). Finally, the mechanical layer of gold is sputtered and patterned to create the beams, Fig. 5(d). In the end, the beams are released by wet etching and supercritical drying, Fig. 5(e).

\section{B. Experimental Setup}

To avoid stiction, the switches were tested in a sealed vacuum chamber kept at 5-8 mTorr. An illustration of the experimental setup is shown in Fig. 6. The vacuum level is sufficient to reduce the moisture in the chamber, but not to ensure a clean gold surface, and thus a thin hydrocarbon layer probably coats the gold surface [21]. Nevertheless, at low moisture, the switches showed significantly reduced adhesion. When operated in air, the switches sometimes stuck down, but those operated in vacuum did not. The chamber is also fitted with a temperature controller that operates from room temperature to over $700 \mathrm{~K}$.

Contact resistance has been shown both experimentally and numerically to remain equal to its dc value at extremely high frequencies (for these switches, higher than $20 \mathrm{THz}$ ) [22], [23]. Hence, our experiments were simplified by measuring the dc contact resistance rather than $\mathrm{S}$-parameters. Two multimeters were used to record current flow and voltage drop across the contact using the four-point probe technique. A dual-channel power supply was used to provide the contact voltage as well as the actuation signal, with a voltage amplifier to provide the high actuation voltage. All instruments were controlled by a computer running LabVIEW. The contact force was controlled by varying the actuation voltage. We found that after fabrication, the switches normally showed very high contact resistance (above $100 \mathrm{k} \Omega$ ). However, by applying a burn-in contact voltage of $2-3 \mathrm{~V}$, the resistance was reduced to 1-2 $\Omega$. The burn-in process is very similar to A-fritting as discussed by Holm [10]. It is unknown, however, whether the same physics is involved. After burn-in, 500 cold-switched break-in cycles were performed.

The power supply (Agilent E3646A) allows both a current limit and a voltage limit to be set. When the output is turned on, the instrument increases the voltage until either limit is reached, allowing for operation in either current-controlled or voltagecontrolled modes. Hence, we were able to specify either the contact voltage or the contact current. For example, when applying the 2-3 V burn-in signal mentioned above, we set the current limit to $1 \mathrm{~mA}$, so that the power supply automatically reduced the voltage when the contact resistance dropped, preventing contact melting. Similarly, during the 500 break-in cycles, the power supply provided $5 \mathrm{~mA}$ of current through the contact in each break-in cycle.

\section{EXPERIMENTAL RESULTS}

First, we tested the response of the contact resistance to externally applied heating. As subsequent results will show, we were able to increase the resistance in a switch by mechanical cycling without the application of current to the contact. Using this method, we raised the resistance in a switch to about $68 \Omega$. Using the thermal stage, we then raised the temperature of the entire 


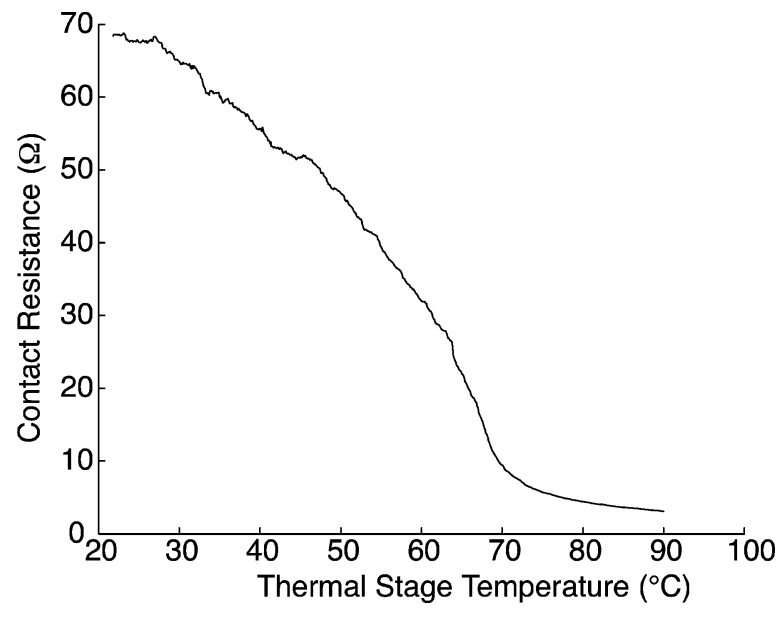

Fig. 7. Contact resistance of a contact heated by a thermal stage.

chip from room temperature to $90{ }^{\circ} \mathrm{C}$ and recorded the contact resistance during heating. The results are shown in Fig. 7. The stage was heated at a rate of $6^{\circ} \mathrm{C}$ per minute, sufficiently slow to assume quasistatic heating of the contact. The contact voltage was maintained below $10 \mathrm{mV}$ to prevent self-heating, and the contact force was approximately $80 \mu \mathrm{N}$. During heating, we observed a large drop in contact resistance, from about $70 \Omega$ to about $3 \Omega$. In fact, the resistance began to drop soon after the temperature began to rise, but the largest portion of the drop occurred between 60 and $70^{\circ} \mathrm{C}$.

A resistance reduction caused by contact heating is called "contact softening" [10], [24]. Note, however, that true contact softening occurs in surfaces heated sufficiently to cause annealing of dislocations in the contact. Since our contacts contain impurities, it is uncertain whether the observed resistance reduction is due to annealing or to enhanced diffusion of the impurities away from the contact at elevated temperature, resulting in a larger contact area. However, since both effects are caused by heating, we will refer to any thermally induced resistance reduction as contact softening. This paper will further explore the cause of the resistance reduction after presenting all of the relevant data.

We can approximate the softening temperature (or temperature causing softening) as $\sim 65{ }^{\circ} \mathrm{C}$, significantly smaller than the published softening temperature of $100{ }^{\circ} \mathrm{C}$, which is based on the assumption of contact annealing. Note that thermal stress during heating will increase the contact force somewhat (predicted contact force at $90{ }^{\circ} \mathrm{C}$ is about $218 \mu \mathrm{N}$ ). However, tests performed at room temperature show that varying contact force by changing actuation voltage causes a $4 \%$ drop in contact resistance between 80 and $218 \mu \mathrm{N}$-much less than the dramatic $95 \%$ decrease seen here. Similarly, several previous results for microscale contact resistance have shown little dependence on contact force for forces between about 100 and 1,000 $\mu \mathrm{N}$ [11], [25], [26]. Hence, the majority of the resistance drop is caused by the externally applied heating.

Next, we studied self-heating of the contact. We measured $V-I$ curves of the contact resistance under varying contact force (that is, using different actuation voltages) for hundreds of contact events on more than 10 switches. The data were taken by stepping the current and measuring the contact voltage. At least 50 cycles separated each $V-I$ test to prevent any test

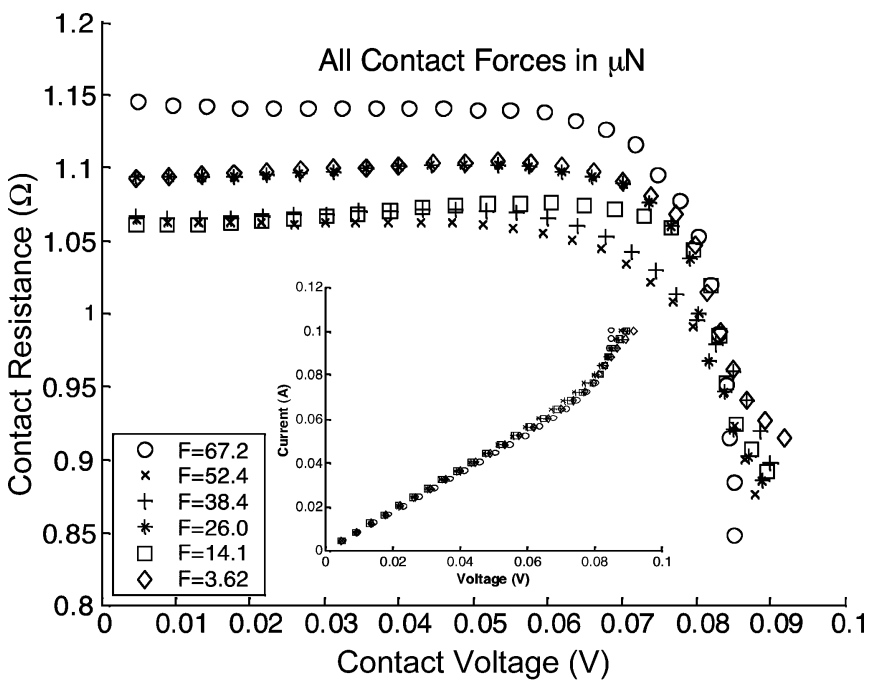

Fig. 8. Voltage vs. contact resistance showing contact softening. The raw $V-I$ data are shown in the inset.

being affected by the previous ones. We found that an increase in contact voltage beyond a threshold caused the contact resistance to decrease in every case. After the decrease, the contact resistance remained low for immediately subsequent cycles. However, during the 50 cycles after each $V-I$ curve, the contact resistance returned to its original value. Fig. 8 shows typical voltage-contact resistance curves for a $500 \mu \mathrm{m}$ beam at six levels of contact force. The raw $V-I$ data are shown in the inset. The resistance remains nearly constant or shows a slight rise (due to the increase in resistivity with temperature) until the voltage reaches approximately $70-80 \mathrm{mV}$. At this point, the resistance decreases rapidly, similar to experimental data in [10] or [27].

\section{A. Low-Resistance Switch Operation}

Experiments also revealed that with switches tested using a low voltage limit of $10 \mathrm{mV}$, the contact resistance tended to continuously increase as the switch cycled. The cause of this resistance increase is not known, but it has been observed previously [1], [3]. We will present a hypothesis for this resistance increase in the next section. However, we found that when the voltage limit was increased above $0.5 \mathrm{~V}$ (while keeping the current below $1 \mathrm{~mA}$ to avoid excessive heating), contact heating caused the contact resistance to stay nearly constant over hundreds of cycles. We observed this behavior under both hot and cold switching. Here, hot switching is defined as switching performed with a voltage placed across the contacts throughout the on-off cycle. In cold switching, voltage is only placed on the switch when the electrodes are in contact. Typical cold-switched results are shown for the both steady resistance and rising resistance in Fig. 9(a) and (b). The contact force in each cycle for this data is about $48 \mu \mathrm{N}$.

To demonstrate that the elevated temperature resulting from contact heating is responsible for avoiding the contact resistance rise, we tested a switch heated to $80^{\circ} \mathrm{C}$ using externallyapplied heating from the thermal stage. The current and voltage limits, and the contact force, were the same as the data in Fig. 9(b) $-1 \mathrm{~mA}, 10 \mathrm{mV}$, and $48 \mu \mathrm{N}$ (note that in this experiment 


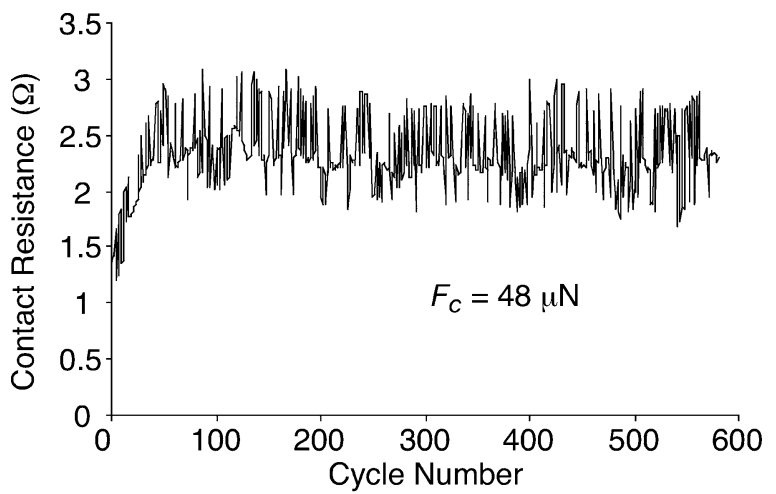

(a)

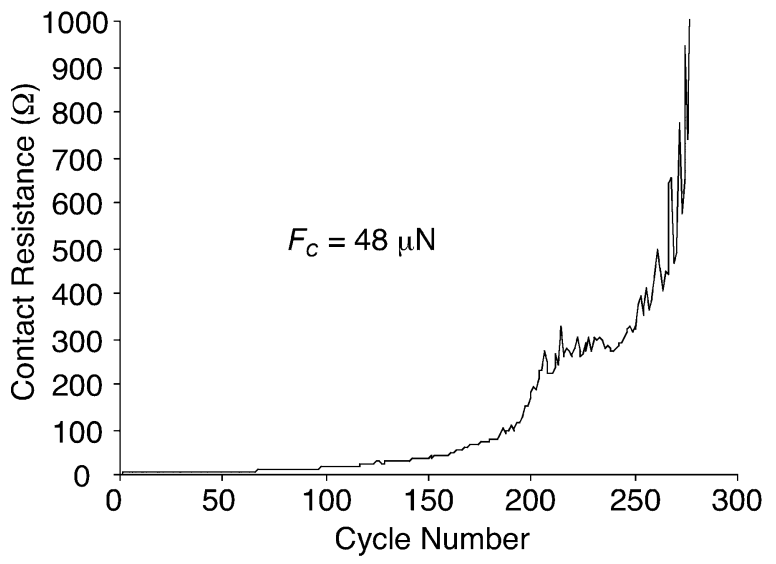

(b)

Fig. 9. Cold-switched operation with current limit of $1 \mathrm{~mA}$ and voltage limit of (a) $1.3 \mathrm{~V}$ (showing steady resistance) and (b) $0.01 \mathrm{~V}$ (showing rising resistance).

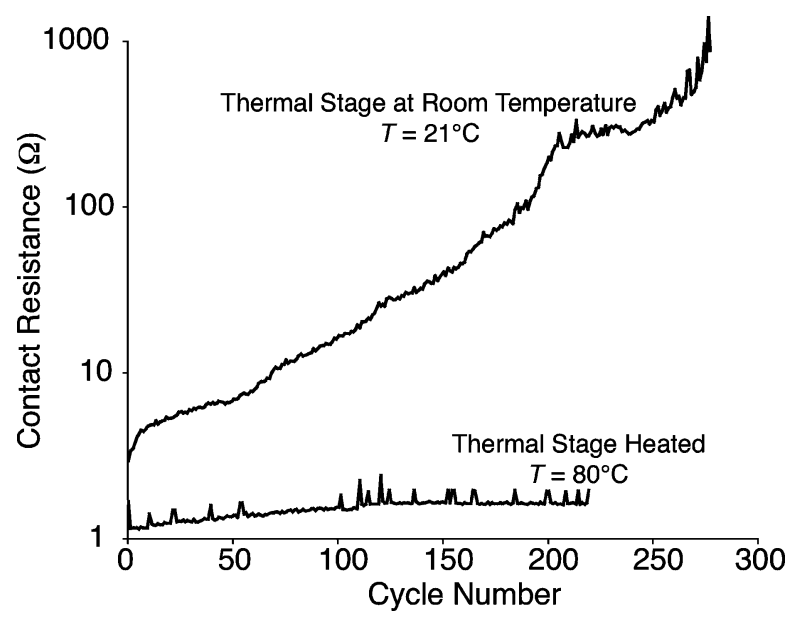

Fig. 10. Comparison of contact resistance for contacts operated at roomtemperature and heated to $80^{\circ} \mathrm{C}$.

the actuation voltage was reduced to keep the contact force the same at elevated temperature). Fig. 10 compares the resulting contact resistance measurements to the data in Fig. 9(b). Over more than 200 cycles, while the room-temperature contact resistance increases hundreds of times, the heated contact resistance remains low, showing that heating prevents the increase in contact resistance. While these low-cycle experiments cannot prove that life may be extended in this way, these results suggest that contact heating may be used to avoid this important failure mechanism for MEMS switches.
TABLE I

EXPERIMENTAL RESULTS REGARDING CONTACT RESISTANCE INCREASE

\begin{tabular}{lcc}
\hline Average Contact Resistance Increase After... & (Ohms) & Std. Dev. \\
\hline 14 days with no operation & 5.24 & \pm 0.222 \\
19 days with no operation & 7.88 & \pm 0.814 \\
100 cycles with $V_{c}=10 \mathrm{~m} V$ & 33.6 & \pm 11.7 \\
100 cycles with $V_{c}=0$ & 36.1 & \pm 7.32 \\
\hline
\end{tabular}

\section{B. Exploration of Resistance Increase}

Table I summarizes experimental results that give a better understanding of contact resistance rise. The first two lines give the average resistance rise after no operation for 14 and 19 days, respectively. In this case, the contact resistance of a switch was measured $(\sim 2 \Omega)$, and the contact was then opened. For the next two weeks, neither it nor any of the switches nearby was tested, while the chip remained in the vacuum chamber. On the fourteenth day, the switch was cycled five times with a contact force of about $48 \mu \mathrm{N}$. The average contact resistance was $5.24 \Omega$ higher than the initial contact resistance, with a standard deviation of $0.2 \Omega$. After five further days of no additional operation, the switch was cycled another five times at the same contact force. In these cycles, the average contact resistance showed an overall increase of $7.88 \Omega$ (with standard deviation of $0.8 \Omega$ ) compared to the initial contact resistance of $2 \Omega$. Hence, although the process was quite slow, the contact resistance increased even when no handling of the switches took place. The most likely cause for such an increase is the gradual build-up or repair of an insulating film.

The last two lines compare the average contact resistance rise after 100 cycles for switches tested with a voltage limit of $10 \mathrm{mV}$ and for switches tested with no contact voltage applied. These experiments were performed to test the hypothesis that electrostatic force pulled impurities into the contact area, increasing resistance. In each case, three sets of 100 cycles were averaged, with each set beginning after the contact was softened to an initial resistance of 5-10 $\Omega$. As before, the contact force in each case was about $48 \mu \mathrm{N}$. The results indicate a statistically insignificant difference. Hence, mechanical cycling alone is an important factor in the resistance increase.

\section{Inconsistencies With Existing Contact Heating Theory}

The data in Fig. 8 indicate contact resistance reduction at a threshold voltage of approximately $75-80 \mathrm{mV}$ for an initial contact resistance near $1 \Omega$. This threshold voltage is called the "softening voltage" [10], [24]. (Initial contact resistance here is the resistance measured at the start, on the linear part of the $V-I$ curve.) However, further testing showed that the softening voltage increases as the contact resistance rises. This was tested by measuring $V-I$ curves with a variety of initial contact resistance magnitudes. Fig. 11 gives the softening voltages extracted from $V-I$ curves of 21 contacts with initial contact resistances varying from 0.5 to $336 \Omega$. The resistances were varied by using mechanical cycling to raise the resistance and contact heating to reduce it. The plot shows that the softening voltage increases for larger initial contact resistance from about $70 \mathrm{mV}$ at $0.5 \Omega$ 


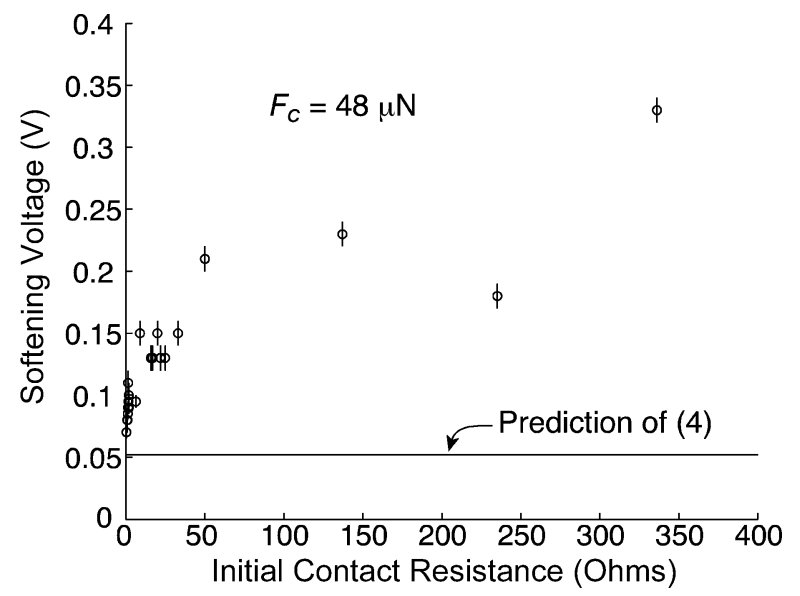

Fig. 11. Softening voltage dependence on contact resistance showing the increase in the softening voltage with higher contact resistance. The lines drawn on each data point give experimental uncertainty in the measurements.

to over $350 \mathrm{mV}$ at $336 \Omega$ (again, a contact force of about $48 \mu \mathrm{N}$ was used). These values are all higher than predictions using existing theory, as shown by the line at $52 \mathrm{mV}$. This line represents the voltage prediction from (4) for heating a contact from 22 to $65^{\circ} \mathrm{C}$, the softening temperature measured from Fig. 7 . In fact, existing theory gives no explanation for why contact heating should depend on the initial contact resistance. To explain these inconsistencies, we develop below improved theory for modeling the contact heating in MEMS-scale contacts. First, we consider further the cause of the resistance increase.

\section{Discussion of Results}

While Fig. 10 shows that contact heating prevents immediate contact resistance rise, the cause of this rapid rise was not apparent. We had previously suggested that cold-working of the contact asperities led to hardening of the metal, increasing contact resistance [28]. However, while this may be responsible for a portion of the observed increase, it is unlikely that strain hardening can account for a thousand-fold increase like that shown in Fig. 9(b). Since contact resistance scales as the square root of hardness [24], such a large increase in resistance would require hardness to increase one million times.

In fact, only the presence of impurities is likely to cause such a large change in contact resistance. We have already mentioned that previous work indicated that sputtered gold films most likely retain a thin insulating layer, probably composed of hydrocarbons adsorbed onto the surface [21]. The high contact resistance of the switches prior to burn-in supports this idea. However, we believe that actual metal-to-metal contact occurs after the initial burn-in because the V-I curves are very linear up to the softening voltage, as will be shown in Fig. 15. In addition, we have found that contacts with lower contact resistance have higher adhesion, suggesting that a larger metal area is in contact [29].

The results reported in Table I further support the idea that the resistance increase is caused by build-up of an insulating film, with mechanical cycling largely responsible for the build-up. The contact behavior suggested by our results is summarized

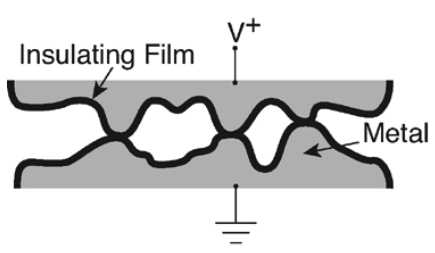

(a)

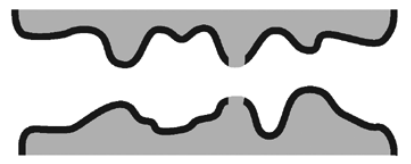

(c)

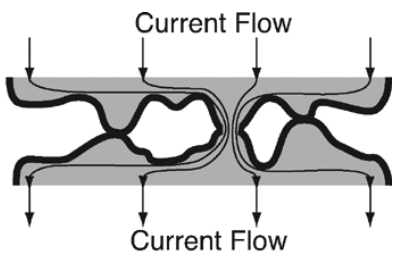

(b)

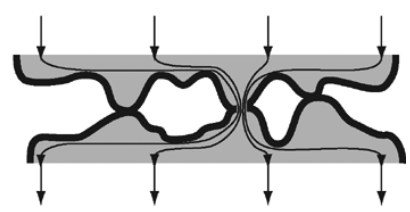

(d)
Fig. 12. Hypothesis describing contact behavior.

in Fig. 12. Part (a) shows two surfaces in contact. Both surfaces are covered with an insulating film. Placing sufficient voltage on the contact surfaces causes breakdown of the insulating film, allowing current flow, Fig. 12(b). When the surfaces pull apart, the contact spot is still bare (no film), as depicted in Fig. 12(c). However, randomness in the contact closing process causes the filmfree spots to be misaligned in subsequent cycles, Fig. 12(d). This causes the insulating film to be pressed onto the edges of the film-free areas, promoting regrowth of the film and leading to increasing contact resistance. This behavior also explains why there appears to be just one real contact spot. When the insulating film initially breaks down in one spot, the voltage immediately drops as current begins to flow, reducing the stress on the rest of the film. Based on this hypothesis, the contact resistance increase would not be observed in ultra-high vacuum, since it has been shown that the film is removed in such an environment [21].

\section{THEORY}

For a contact radius on the order of the electron mean free path (about $38 \mathrm{~nm}$ in gold [30]) or smaller, the current is constricted by both lattice scattering and boundary scattering of electrons. For both ohmic constriction and boundary scattering, the contact resistance $R_{c}$ for a spot of radius $a$ is [31]

$$
R_{c}=\gamma\left(\frac{\lambda}{a}\right) R_{M}+R_{S}=\frac{1+0.83\left(\frac{\lambda}{a}\right)}{1+1.33\left(\frac{\lambda}{a}\right)} \frac{\rho}{2 a}+\frac{4 \rho \lambda}{3 \pi a^{2}}
$$

where $\lambda$ is the mean free path, and $\rho$ is the electrical resistivity. $R_{M}$ is the Maxwell spreading resistance (the resistance due to lattice scattering), and $R_{S}$ is the Sharvin resistance (the additional resistance due to boundary scattering in small constrictions). Also, $\gamma(\lambda / a)$ is a scaling function. While this equation is well-known, most of the existing work on contact heating considers only the contribution of the Maxwell spreading resistance [10], [32]. Even for the smallest contact resistance in Fig. 11 of $0.5 \Omega$, (5) gives a contact radius of $51.8 \mathrm{~nm}$, comparable to the electron mean free path. (For this calculation, we used a measured resistivity of $3.6 \times 10^{-8} \Omega \cdot \mathrm{m}$.) Hence, much of the measured resistance results from boundary scattering of electrons. However, boundary-scattered electrons do 


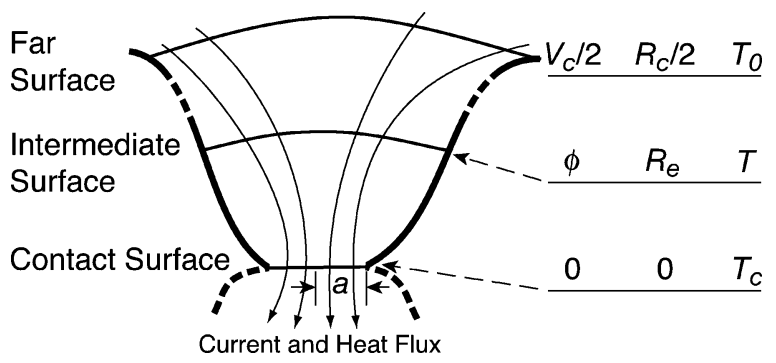

Fig. 13. Half of a contact showing the contact surface, an intermediate isothermal-equipotential surface, and a surface far from the contact spot. Labels show the potential and resistance (measured with respect to the contact surface) as well as temperature.

not transfer heat to the metal lattice within the contact constriction (see [33]), leading to a reduction in the contact temperature for a given contact voltage. The existing model of contact heating does not include this effect.

In addition, the existing model of (4) assumes that the temperature far from the contact spot is equal in each of the contacting bodies. This is not true for many MEMS contacts, since the small size of the moving contact makes heating of the entire moving body unlikely. Hence, a difference exists between the substrate (at room temperature) and the moving body (at an elevated temperature). Therefore, understanding of the nanoscale contact heating requires consideration of both the device-level temperature and the extremely localized heating of the contact spot. At the nanometer scale, models relate the real contact size and contact voltage with the contact temperature. On the device level, an integrated electrothermal model is necessary to describe the relationship between current flow and temperature.

\section{A. Asperity Heating Model}

The goal of the nanoscale contact heating model is to relate the contact voltage $V_{c}$ to the contact spot temperature $T_{c}$ while considering the effects of contact spot size. The principle difference between the theory presented here and existing theory is that we assume that heating in the contact is due only to the Maxwell term in (5), $\gamma R_{M}$.

Greenwood and Williamson have previously shown that equipotential surfaces are also isothermals in a contact [32]. For initial development of the model, we assume that the contact is symmetric, with maximum temperature at the contact asperity. This assumption will be relaxed later. Hence, we analyze a half-contact. See Fig. 13 for an illustration, with labels showing potential, resistance, and temperature at the contact spot, an arbitrary intermediate isothermal-equipotential surface, and on a surface sufficiently far from the contact. We further assume that the potential $\phi$ on an equipotential surface can be broken into Maxwell and Sharvin components as

$$
\phi=\phi_{M}+\phi_{S}=I\left(R_{e M}+R_{e S}\right)
$$

$R_{e M}$ and $R_{e S}$ are the Maxwell and Sharvin components of resistance between the surface and the contact spot, and $R_{e}=$ $R_{e M}+R_{e S}$ is the total resistance in the same volume.

Assuming that the Maxwell resistance is the only source of contact heating, we can write the total heat generated between the contact spot and any isothermal as $I \phi_{M}$. The isothermal differential temperature is then

$$
\mathrm{d} T=-I \phi_{M} \mathrm{~d} R_{t}
$$

where $\mathrm{d} R_{t}$ is the corresponding differential thermal resistance of the surface. If we assume that conduction through the metal is the dominant form of heat transfer through the contact, we can also compare the differential thermal and electrical resistances on any equipotential surface via the relation

$$
\mathrm{d} R_{t}=\frac{\mathrm{d} R_{e}}{\rho_{e} \kappa_{e}}=\frac{\mathrm{d} \phi}{I \rho_{e} \kappa_{e}} .
$$

Here, $\rho_{e}$ and $\kappa_{e}$ are the effective electrical resistivity and thermal conductivity of the metal accounting for size effects. Substituting (6) and (8) into (7) then gives

$$
\mathrm{d} T=-\frac{I R_{e M}}{\rho_{e} \kappa_{e}} \mathrm{~d} \phi=-\frac{R_{e M} \phi}{R_{e} \rho_{e} \kappa_{e}} \mathrm{~d} \phi .
$$

Integrating from the contact spot to the far surface produces

$$
\int_{T_{0}}^{T_{c}} \rho_{e} \kappa_{e} \mathrm{~d} T=\int_{0}^{\frac{V_{c}}{2}} \frac{R_{e M} \phi}{R_{e}} \mathrm{~d} \phi .
$$

Unfortunately, the detailed geometry of the contact is required to calculate $R_{e}$ and $R_{e M}$. However, we may estimate the ratio $R_{e M} / R_{e}$ as that of the overall Maxwell resistance to the overall contact resistance, $R_{e M} / R_{e} \approx \gamma R_{M} / R_{c}$. Making this substitution into (10) and evaluating the integral gives

$$
\int_{T_{0}}^{T_{c}} \rho_{e} \kappa_{e} \mathrm{~d} T=\frac{\gamma R_{M}}{8 R_{c}} V_{c}^{2} .
$$

The left-hand side of (11) may be evaluated using the Wiedemann-Franz law. This law states that for metals, $\rho \kappa=L T$, where $\rho$ and $\kappa$ are the material electrical resistivity and thermal conductivity [34]. The Wiedemann-Franz law has been shown to apply even at atomistic length scales, the size of the smallest possible contact spots, so it applies to the factor $\rho_{e} \kappa_{e}$ as well [35], resulting in

$$
L\left(T_{c}^{2}-T_{0}^{2}\right)=\frac{\gamma R_{M}}{4 R_{c}} V_{c}^{2} .
$$

The only difference between (12) and (4) is the factor $\gamma R_{M} / R_{c}$. This factor is nearly unity when $R_{c}$ is small (when $a$ is much larger than $\lambda$ ), and it decreases to nearly zero for large $R_{c}$ (when $a$ is much smaller than $\lambda$ ). Therefore, for a small contact resistance, (12) is equal to (4), deviating only when boundary scattering contributes to the contact resistance.

Equation (12) gives the asperity temperature assuming both contact surfaces are at the same temperature $T_{0}$. As described above, the moving surface in a MEMS switch is likely to be heated by the passage of current, and so the material in the contact surfaces is at different temperatures $T_{2}$ and $T_{1}$ (we arbitrarily choose $T_{2}>T_{1}$ ). In this case, a constant additional heat 
flux $\dot{q}$ will flow through the contact, and so (7) will have an additional term

$$
\mathrm{d} T=\left(-I \phi_{M} \pm \dot{q}\right) \mathrm{d} R_{t} .
$$

The flux $\dot{q}$ is added if the isothermal surface is in contact body 1 (at $T_{1}$ ) and subtracted in body 2 (at $T_{2}$ ). Following the same derivation used for (12) leads to

$$
\begin{aligned}
& L\left(T_{c}^{2}-T_{1}^{2}\right)=\frac{\gamma R_{M}}{4 R_{c}} V_{c}^{2}+\frac{\dot{q}}{I} V_{c} \\
& L\left(T_{c}^{2}-T_{2}^{2}\right)=\frac{\gamma R_{M}}{4 R_{c}} V_{c}^{2}-\frac{\dot{q}}{I} V_{c} .
\end{aligned}
$$

Eliminating $\dot{q} V_{c} / I$ from both equations yields

$$
T_{c}^{2}=\frac{\gamma R_{M}}{4 R_{c} L} V_{c}^{2}+\frac{1}{2}\left(T_{1}^{2}+T_{2}^{2}\right) .
$$

We remark that (16) is identical to (12) when $T_{1}=T_{2}=T_{0}$. We also note that (12) has been experimentally validated using MEMS switch contacts [36].

\section{B. Electrothermal Model}

Use of (16) requires knowledge of $T_{1}$ and $T_{2}$, the temperatures of the contacting bodies. We can assume that the fixed contact remains at ambient temperature, $T_{1} \approx T_{0}$. However, electrothermal modeling of the switch is required to calculate $T_{2}$, the temperature in the moving contact. The electrothermal model used here is described in [37]. Briefly, 2-D finite element modeling (FEM) is used to solve the heat equation with the electric current as a heat source. The model includes effects due to heat conduction and contact heating, as well as electrical and thermal contact resistance. Convective and radiative heat transfer are ignored because they are insignificant.

\section{Comparison to Experimental Data}

Using the combination of FEM and (16), we analyzed the data of Fig. 8 to determine the contact spot temperature and percent real contact area increase. First, we used the FEM to calculate the temperature in the beam near the contact. We then computed the contact spot temperature using (16). Finding the real contact area requires knowledge of the change in average electrical resistivity as the contact is heated. Holm estimated that the average resistivity changes as [10]

$$
\bar{\rho}=\rho_{0}+\frac{2 L \Delta T}{3 \kappa}
$$

where $\bar{\rho}$ and $\rho_{0}$ are respectively the average and room temperature resistivities and $\Delta T=T_{c}-T_{0}$, the difference between the contact spot temperature and room temperature. We estimated the contact spot size for each data point of Fig. 8 using (5) and (17), allowing estimation of the area increase as $a^{2} / a_{0}^{2}$, where $a$ is the real contact radius and $a_{0}$ is the initial contact radius (the first data point for each $V-I$ curve).

Fig. 14 shows the calculated percent contact area increase as a function of the predicted contact temperature. The data show rapidly increasing contact area above about $65^{\circ} \mathrm{C}$. This result agrees well with the experimental threshold temperature

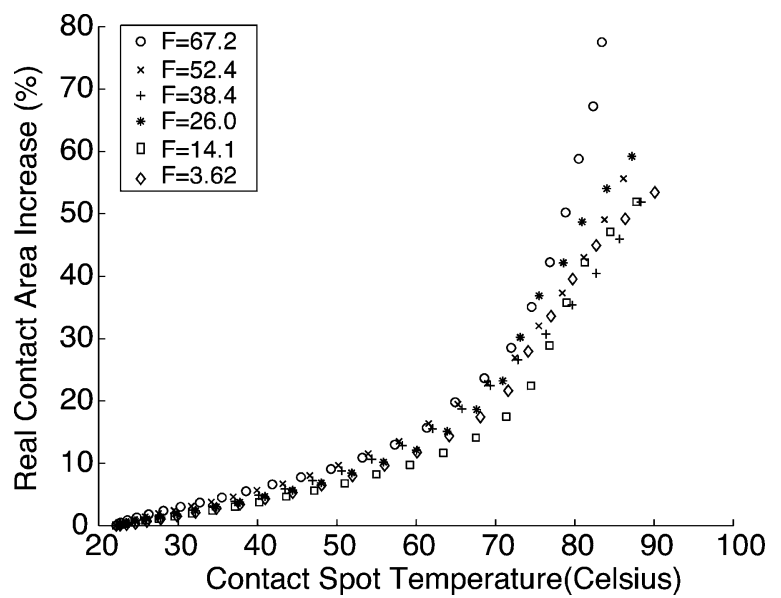

Fig. 14. Percent increase in contact area as a function of contact spot temperature for the data shown in Fig. 8.

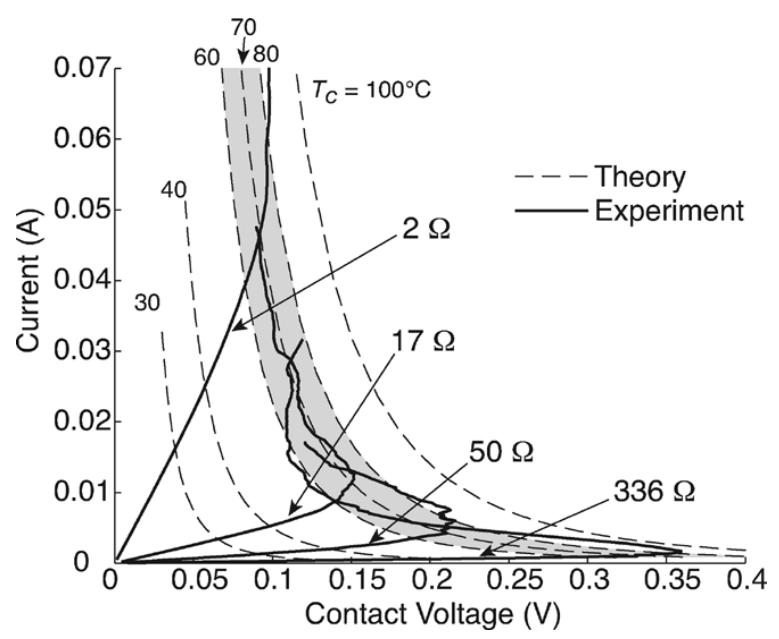

Fig. 15. Four V-I curves with contact temperature isothermals. The expected softening region, $60-80^{\circ} \mathrm{C}$, is shaded for emphasis. After becoming nonlinear, the $V-I$ curves follow the shaded isothermals, showing that softening continues at the same temperature as current increases.

of $60-70{ }^{\circ} \mathrm{C}$. However, we emphasize again that these temperatures are well below the published softening temperature for gold of $100{ }^{\circ} \mathrm{C}$ [10]. We believe that the resistance decrease in our experiments is due to the thermal breakdown of bonds between the gold and the insulating film, allowing the film to be easily pushed aside. Alternatively, it has been shown that gold melting temperature drops for small gold particles [38]. Hence, it is also possible that softening occurs at reduced temperature for small contact spots. Softening at the published softening temperature has been linked to annealing, leading to a reduction in hardness of the work-hardened contact spot [10]. We believe that the reason for the disparity is that our measurements are recording a different physical phenomenon (breakdown of an insulating film) that seems to dominate contact resistance in low-force MEMS contacts.

Four V-I curves from the tests summarized in Fig. 11 are shown in Fig. 15 and compared to model predictions of asperity temperature. The contacts had initial resistance of $2,17,50$, and $336 \Omega$. Contact temperature isothermals are shown for temperatures from 30 to $100^{\circ} \mathrm{C}$. These isothermal lines were calculated 


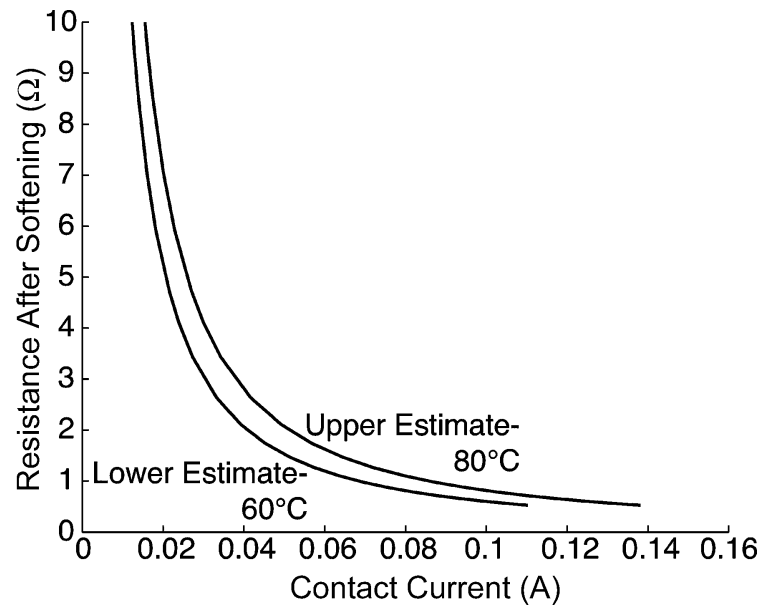

Fig. 16. The expected after-softening contact resistance as a function of current carried by the contact.

by converting (16) into two parametric equations describing the voltage and current in a contact at a given temperature

$$
\begin{aligned}
V_{c} & =\sqrt{\frac{2 R_{c} L}{\gamma R_{M}}\left(2 T_{c}^{2}-T_{1}^{2}-T_{2}^{2}\right)} \\
I & =\frac{V_{c}}{R_{c}} .
\end{aligned}
$$

The isothermals in Fig. 15 were generated by choosing a range of values for the contact resistance $R_{c}$, setting $T_{1}$ equal to the ambient temperature of $22^{\circ} \mathrm{C}$, and calculating $T_{2}$ using the finite element model. The onset of nonlinearity for each $\mathrm{V}$-I curve occurs at approximately $70^{\circ} \mathrm{C}$, agreeing with the experimentally determined softening temperature. Moreover, a key result is the nonlinear part of the $V-I$ curves. Once the $V-I$ lines become nonlinear, they follow the isothermals, staying largely within the shaded region $\left(60-80{ }^{\circ} \mathrm{C}\right)$. Therefore, when the current is increased after the first contact softening, the contact resistance continues to decrease, keeping the contact temperature approximately constant. This important result provides another strong validation of the theory.

The $V-I$ data in Fig. 15 are similar to data for B-fritting presented by Holm [10]. B-fritting is the growth of a contact spot due to breakdown of an insulating film under sufficient voltage. Our results suggest that in this case, B-fritting is caused by heating of the contact spot-an idea supported also by the data of Fig. 7, when the switch was heated externally by the thermal stage. By contrast, Holm attributed B-fritting to electrostatic force acting on ions in the film.

Fig. 15 also illustrates another important result. Since the $V-I$ curve after softening stays near the $60-80{ }^{\circ} \mathrm{C}$ isothermals, we may predict the reduced resistance resulting from softening as a function of the current carried by the contact. The resistance after softening is simply that corresponding to a contact temperature of roughly $60-80^{\circ} \mathrm{C}$. Fig. 16 shows the resulting relationship. The upper and lower estimates on this plot represent the isothermal curves corresponding to 80 and $60{ }^{\circ} \mathrm{C}$, respectively, in Fig. 15. The isothermal curves are replotted to illustrate the relationship between the contact resistance after softening and the current causing softening. This understanding is vital in modeling the contact behavior after heating has taken place.

\section{CONCLUSION}

We performed experiments that demonstrated a contact resistance reduction when a metal-to-metal contact is heated. This reduction is called contact softening. Softening occurs both for externally-heated contacts and for those heated by the passage of current (see Figs. 7 and 8). We also found that existing theory over-predicts internal heating for MEMS contacts. In addition, we showed that contacts with larger resistance require a higher contact voltage for heating. This effect is not predicted by existing theory (see Fig. 11). Therefore, we proposed a new approach to explain these effects. The resulting theory predicts that for a given contact voltage, small contact spots with a radius less than about $40 \mathrm{~nm}$ (those with high resistance) will show reduced heating compared to larger spots. This is because boundaryscattered electrons (which account for much of the resistance of small spots) do not heat the contact region. Our predictions compare well with experimental data. Using the theory to explain experimental results, we can confirm that both externally-heated and voltage-heated contacts are softened at a temperature of $60-70{ }^{\circ} \mathrm{C}$. Further, as shown in Fig. 15, increasing the contact current after softening takes place leads to further resistance reduction. This further reduction occurs such that the contact temperature remains nearly constant despite the increased current (see the shaded region in Fig. 15). This allows approximate prediction of the contact resistance after the contact has been softened at a given current.

We also found that contact heating can be used to control contact resistance increase, a commonly-reported failure mechanism for MEMS switches. Our experiments showed that unheated contacts showed significantly larger resistance as the switch cycled. The data suggest that this increase may be due to the build-up of an insulating film. However, contacts heated either externally or internally (by the passage of current) showed low resistance over many hundreds of cycles (see Figs. 9 and 10). Further experiments are planned to study the effect of contact heating on switch lifetime. We believe that heating breaks down the insulating film, reducing resistance. Therefore, contact heating allows control of contact resistance in MEMS switches. Comparisons between theory and experiments verify this conclusion.

\section{ACKNOWLEDGMENT}

The authors would like to thank R. Webbink for his suggestions on mechanical modeling of MEMS switches.

\section{REFERENCES}

[1] S. Majumder, N. E. McGruer, G. G. Adams, P. M. Zavracky, R. H. Morrison, and J. Krim, "Study of contacts in an electrostatically actuated microswitch," Sens. Actuators A: Phys., vol. 93, pp. 19-26, 2001.

[2] J. B. Muldavin and G. M. Rebeiz, "Inline capacitive and DC-contact MEMS shunt switches," IEEE Microwave Wireless Compon. Lett., vol. 11, no. 8, pp. 334-336, Aug. 2001.

[3] R. Chan, R. Lesnick, D. Becher, and M. Feng, "Low-actuation voltage RF MEMS shunt switch with cold switching lifetime of seven billion cycles," J. Microelectromech. Syst., vol. 12, no. 5, pp. 713-719, Oct. 2003.

[4] G. M. Rebeiz and J. B. Muldavin, "RF MEMS switches and switch circuits," IEEE Microw. Mag., vol. 2, no. 4, pp. 59-71, Dec. 2001.

[5] G. M. Rebeiz, RF MEMS, Theory, Design, and Technology. New York: Wiley, 2003. 
[6] J. Lampen, S. Majumder, R. Morrison, A. Chaudhry, and J. Maciel, "A wafer-capped, high-lifetime ohmic MEMS RF switch," Int. J. RF Microwave Computer-Aided Eng., vol. 14, no. 4, pp. 338-344, Jul. 2004

[7] B. J. Gally, C. C. Abnet, and S. Brown, "Investigation of wear of microelectromechanical contacts," in Proc. Mat. Res. Soc. Symp., vol. 605, 2000, pp. $117-122$.

[8] E. J. J. Kruglick and K. S. J. Pister, "Lateral MEMS microcontact considerations," J. Microelectromech. Syst., vol. 8, no. 3, pp. 264-271, Sept. 1999.

[9] M. Hasegawa, T. Yamamoto, and K. Sawa, "Significant increase of contact resistance of silver contacts by mechanical switching actions," IEEE Trans. Comp., Hybrids, Manufact. Technol., vol. 15, no. 2, pp. 177-183, 1992.

[10] R. Holm, Electric Contacts-Theory and Applications, 4th ed. Berlin, Germany: Springer-Verlag, 1967.

[11] D. Hyman and M. Mehregany, "Contact physics of gold microcontacts for MEMS switches," IEEE Trans. Comp. Packag. Technol., vol. 22, no. 3, pp. 357-364, Sep. 1999.

[12] X. Yan, N. E. McGruer, G. G. Adams, and S. Majumder, "Finite element analysis of the thermal characteristics of mems switches," in Proc. 12th Int. Conf. on Transducers, Solid-State Sensors, Actuators, and Microsystems, Jun. 2003.

[13] B. B. Mandelbrot, The Fractal Geometry of Nature. New York: Freeman, 1983.

[14] W. Yan and K. Komvopoulos, "Contact analysis of elastic-plastic fractal surfaces," J. Appl. Phys., vol. 84, no. 7, pp. 3617-3624, 1998.

[15] S. Majumder, N. E. McGruer, P. M. Zavracky, G. G. Adams, R. H. Morrison, and J. Krim, "Measurement and modeling of surface micromachined, electrostatically actuated microswitches," in Proc. 1997 Int. Conf. Solid-State Sensors and Actuators, Jun. 1997, pp. $1145-1148$.

[16] B. Kracke and B. Damaschke, "Measurement of nanohardness and nanoelasticity of thin gold films with scanning force microscope," Appl. Phys. Lett., vol. 77, no. 3, pp. 361-363, Jul. 2000.

[17] H. D. Espinosa and B. C. Prorok, "Size effects on the mechanical behavior of gold thin films," J. Mat. Sci., vol. 38, pp. 4125-4128, 2003.

[18] H. D. Espinosa, B. C. Prorok, and M. Fischer, "A methodology for determining mechanical properties of freestanding thin films and MEMS materials," J. Mechan. Phys. Solids, vol. 51, pp. 47-67, 2003.

[19] F. E. Gardiol, Microstrip Circuits. New York: Wiley, 1994.

[20] B. D. Jensen, "Multi-Physics Modeling and Experimental Investigation of Low-Force MEMS Switch Contact Behavior," Ph.D. dissertation, University of Michigan, Ann Arbor, MI, 2004.

[21] J. W. Tringe, T. A. Uhlman, A. C. Oliver, and J. E. Houston, "A single asperity study of Au/Au electrical contacts," J. Appl. Phys., vol. 93, no. 8, pp. 4661-4669, 2003.

[22] R. Kwiatkowski, M. Vladimirescu, A. Zybura, and S. Choi, "Scattering parameter model of low level electrical contacts in electro-mechanical microwave switches-a switch manufacturer approach," in Proc. 48th IEEE Holm Conf. on Electrical Contacts, 2002, pp. 221-230.

[23] J. D. Lavers and R. R. Timsit, "Constriction resistance at high signal frequency," IEEE Trans. Comp. Packag. Technol., vol. 25, no. 3, pp. 446-452, Sep. 2002.

[24] R. S. Timsit, "Electrical contact resistance: properties of stationary interfaces," IEEE Trans. Comp. Packag. Technol., vol. 22, pp. 85-98, 1999.

[25] J. Schimkat, "Contact materials for microrelays," in Proc. 11 th IEEE Int. Conf. on Microelectromech. Syst., 1998, pp. 190-194.

[26] B. L. Pruitt, W.-T. Park, and T. W. Kenny, "Measurement system for low force and small displacement contacts," J. Microelectromech. Syst., vol. 13, no. 2, pp. 220-229, Apr. 2004

[27] F. P. Bowden and J. B. P. Williamson, "Electrical conduction in solids. I. Influence of the passage of current on the contact between solids," Proc. Roy. Soc. London. Series A, Math. Phys. Sci., vol. 246, no. 1244, pp. 1-12, Jul. 1958

[28] B. D. Jensen, K. Huang, L. W. Chow, K. Saitou, J. L. Volakis, and K. Kurabayashi, "Asperity heating for repair of metal contact RF MEMS switches," in Proc. 2004 IEEE MTT-S International Microwave Symposium Digest, vol. 3, 2004, pp. 1939-1942.

[29] B. D. Jensen, L. L.-W. Chow, J. L. Volakis, and K. Kurabayashi, "Adhesion effects on contact opening time in MEMS switches," in Proc. ASME/STLE Int. Joint Tribology Conf., 2004, Paper no. TRIB2004-64 350.

[30] N. W. Ashcroft, N. D. Mermin, and D. Mermin, Solid State Physics, 1st ed. New York: Holt, Rinehart and Winston, 1976.

[31] B. Nikolić and P. B. Allen, "Electron transport through a circular constriction," Phys. Rev. B, vol. 60, no. 6, pp. 3963-3969, 1999.
[32] J. A. Greenwood and J. B. P. Williamson, "Electrical conduction in solids. II. Theory of temperature-dependent conductors," Proc. Roy. Soc. London. Series A, Math. Phys. Sci., vol. 246, no. 1244, pp. 13-31, 1958.

[33] L. Weber, M. Lehr, and E. Gmelin, "Investigation of the transport properties of gold point contacts," Phys. B: Condensed Matter, vol. 217, pp 181-192, 1996.

[34] C. Kittel, Introduction to Solid State Physics, 6th ed. New York: John Wiley \& Sons, Inc., 1986.

[35] S. Ciraci, A. Buldum, and I. P. Batra, "Quantum effects in electrical and thermal transport through nanowires," J. Phys. Condensed Matter, vol 13, pp. R537-R568, 2001

[36] B. D. Jensen, K. Huang, L. W. Chow, and K. Kurabayashi, "Low-force contact heating and softening using micromechanical switches in diffusive-ballistic electron transport transition," Appl. Phys. Lett., vol. 86 2005.

[37] B. D. Jensen, K. Saitou, J. Volakis, and K. Kurabayashi, "Fully integrated electrothermal multidomain modeling of RF MEMS switches," IEEE Microwave Wireless Compon. Lett., vol. 13, no. 9, pp. 364-366, Sep. 2003.

[38] P. Buffat and J.-P. Borel, "Size effect on the melting temperature of gold particles," Phys. Rev. A, vol. 13, no. 6, pp. 2287-2298, Jun. 1976

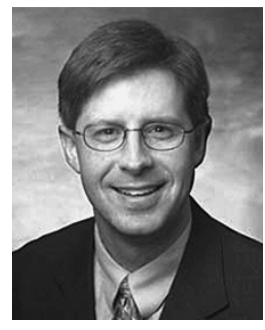

Brian D. Jensen received the Ph.D. degree in mechanical engineering, as well as the M.S. degree in electrical engineering, from the University of Michigan, Ann Arbor, in 2004. He also received the B.S. and M.S. degrees in mechanical engineering from Brigham Young University, Provo, UT, in 1996 and 1998, respectively.

He has served as an Assistant Professor in the Mechanical Engineering Department at Brigham Young University since January 2005. He was previously a Postdoctoral Fellow at the University of Michigan. Before pursuing his Ph.D., he worked at Sandia National Laboratories as a micromechanical designer. As a graduate student, he was the recipient of a $\mathrm{Na}-$ tional Science Foundation Graduate Research Fellowship as well as a National Defense Science and Engineering Graduate Fellowship. He holds seven U.S. patents and has authored or coauthored over 35 technical papers.

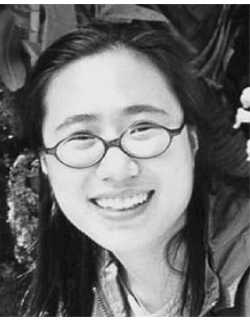

Linda L.-W. Chow (S'02) is currently working towards the $\mathrm{Ph} . \mathrm{D}$. degree in mechanical engineering at University of Michigan, Ann Arbor. She has studied RF MEMS switch design, fabrication process development, and thermal/contact reliability studies since fall 2002. In her master studies, she developed a low temperature fabricated moisture sensor for packaging reliability study, which was awarded as the Best Student Paper in IEEE EMAP2000.

She also worked as a teaching assistant for two semesters, which were both chosen as the Best

Teaching Assistant.

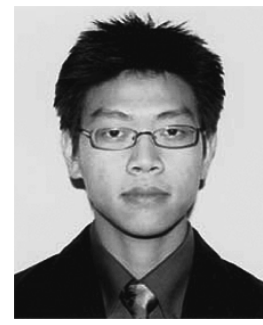

Kuangwei Huang is an undergraduate senior from the University of Michigan, Ann Arbor, and received the B.S. degree in mechanical engineering in May 2005.

He has worked as a Research Assistant in the Department of Mechanical Engineering's Microelectromechanical Systems Testing Laboratory for eight months, studying the contact heating of contact-type RF MEMS switches.

Mr. Huang is a Member of the Mechanical Engineering Honor Society, Pi Tau Sigma, a recipient of the James B. Angell Scholar Award, and he is recognized in the university's Dean's List. 


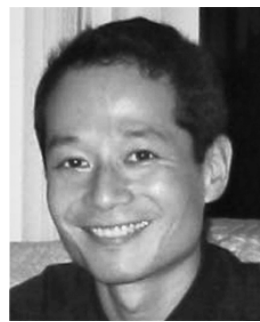

Kazuhiro Saitou (M'97) received the Ph.D. degree in mechanical engineering from the Massachusetts Institute of Technology (MIT), Cambridge, in 1996.

From 1997 to 2003, he was an Assistant Professor with the Department of Mechanical Engineering, University of Michigan, Ann Arbor, where he is currently an Associate Professor. His research interests include design automation and optimization, design for $\mathrm{X}$, where $\mathrm{X}=$ manufacture, assembly, robustness, environment, modeling and optimization of microelectromechanical systems, and evolutionary computation in mechanical design.

Dr. Saitou is a Member of American Society of Mechanical Engineers, Society of Manufacturing Engineers, Association for Computing Machinery, and Sigma Xi. He currently serves as an Associate Editor for IEEE TRANSACTIONS ON AUTOMATION SCIENCE AND ENGINEERING and an editorial board member of the International Journal of CAD/CAM, and Genetic Programming and Evolvable Machines. He was the recipient of the 1999 CAREER Award from the National Science Foundation, and of the Best Paper Award at the 5th International Symposium on Tools and Methods of Competitive Engineering in 2004.

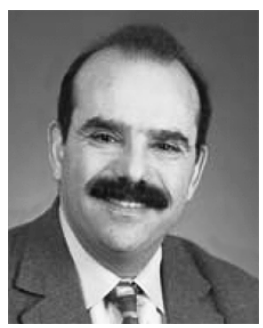

John L. Volakis (S'77-A'79-S'80-M'82-SM'88F'96) was born on May 13, 1956 in Chios, Greece, and immigrated to the United States in 1973. He received the B.E. degree (summa cum laude), in 1978 from Youngstown State University, Youngstown, $\mathrm{OH}$, and the M.Sc. and Ph.D. degreesfrom the Ohio State University, Columbus, OH, in 1979 and 1982, respectively.

From 1982 to 1984, he was with Rockwell International, Aircraft Division (now Boeing Phantom Works), Lakewood, CA, and during 1978-1982, he was a Graduate Research Associate at the Ohio State University ElectroScience Laboratory. He has been a Professor in the Electrical Engineering and Computer Science Department at the University of Michigan, Ann Arbor, since 1984. He also served as the Director of the Radiation Laboratory from 1998 to 2000. In January 2003, he was the Roy and Lois Chope Chair Professor of Engineering at the Ohio State University, Columbus, and also serves as the Director of the ElectroScience Laboratory. His primary research deals with computational methods, electromagnetic compatibility and interference, design of new RF materials, multiphysics engineering, and bioelectromagnetics. He has published over 220 articles in major refereed journal articles (nine of these have appeared in reprint volumes), nearly 300 conference papers and 10 book chapters. In addition, he coauthored two books: Approximate Boundary Conditions in Electromagnetics (Institution of Electrical Engineers, London, 1995) and Finite Element Method for Electromagnetics (IEEE Press, New York, 1998). He has also written well-edited coursepacks and delivered short courses on numerical methods, antennas and frequency selective surfaces. In 1998, he received the University of Michigan College of Engineering Research Excellence award and in 2001, he received the Department of Electrical Engineering and Computer Science Service Excellence Award. He is listed by ISI among the top 250 most referenced authors in Computer Science/Engineering (2004); He graduated/mentored nearly $40 \mathrm{Ph} . \mathrm{D}$. students/postdoctorals, and coauthored with them five best paper awards (four at IEEE conferences and one at the Antenna Measurement Techniques Association).

Dr. Volakis served as an Associate Editor of the IEEE TRANSACTIONS ON ANTENNAS AND PROPAGATION from 1988 to 1992; as an Associate Editor of Radio Science from 1994 to 1997; Chaired the 1993 IEEE Antennas and Propagation Society Symposium and Radio Science Meeting, and was a member of the AdCom for the IEEE Antennas and Propagation Society (1995-1998). $\mathrm{He}$ was the 2004 President of the IEEE Antennas and Propagation Society, and serves as the Technical Chair for USNC/URSI and as anAassociate Editor for the Journal Electromagnetic Waves and Applications, the IEEE ANTENNAS AND Propagation SOCIETY MAGAZINE, and the URSI Bulletin. He is a member of Sigma Xi, Tau Beta Pi, Phi Kappa Phi, and Commission B of URSI. He is also listed in several Who's Who directories, including Who's Who in America.

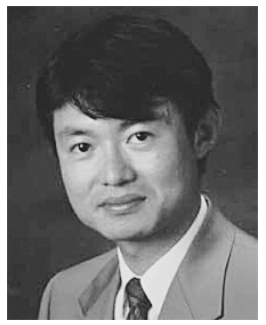

Katsuo Kurabayashi (M'00) received the B.S. degree in precision engineering from the University of Tokyo, Japan, in 1992, and the M.S. and Ph.D. degrees in materials science and engineering with electrical engineering minor from Stanford University, CA, in 1994 and 1998, respectively.

His dissertation work focused on measurement and modeling of the thermal transport properties of electronic packaging and organic materials for integrated circuits under the contract with the Semiconductor Research Corporation (SRC). Upon completion of his Ph.D. program, he was hired as Research Associate with the Department of Mechanical Engineering at Stanford University for 12 months. In January 2000, he joined the faculty of the University of Michigan, Ann Arbor, where he is currently Assistant Professor of Mechanical Engineering and Electrical Engineering and Computer Science. His group at Michigan studies multiphysics modeling and characterization of RF MEMS, biomolecular motor hybrid NEMS/MEMS technology, and polymer-on-silicon strain-tunable photonic devices.

Dr. Kurabayashi is a recipient of the Semiconductor Research Corporation (SRC) Best Paper Award (1998), the NSF CAREER Award (2001), and the University of Michigan Robert Caddell Memorial Award (2004). 